\title{
Mitochondrial DNA profiling reveals two lineages of sun bears in East and West
}

Malaysia

Wai-Ling Lai ${ }^{\mathrm{a}}$, Jactty Chew $^{\mathrm{a}}$, Derek Gatherer ${ }^{\mathrm{b}}$, Dusit Ngoprasert $^{\mathrm{c}}$, Sadequr Rahman ${ }^{\mathrm{d}, \mathrm{e}}$, Qasim Ayub $^{\text {d,e }}$, Adrian Kannan ${ }^{\mathrm{a}}$, Eleanor Vaughan ${ }^{\mathrm{b}}$, Siew Te Wong ${ }^{\mathrm{f}}$, Noor Azleen Mohd Kulaimi ${ }^{\mathrm{g}}$ \& Shyamala Ratnayeke ${ }^{\mathrm{a} *}$

${ }^{a}$ Department of Biological Sciences, Faculty of Science and Technology, Sunway University, No. 5, Jalan Universiti, Bandar Sunway, 47500 Selangor Darul Ehsan, Malaysia.

${ }^{\mathrm{b}}$ Division of Biomedical \& Life Sciences, Faculty of Health \& Medicine, Lancaster University, Lancaster, United Kingdom.

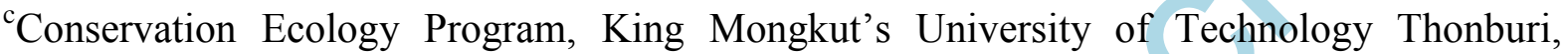
Bangkok, Thailand

${ }^{\mathrm{d}}$ Monash University Malaysia Genomics Facility, School of Science, Jalan Lagoon Selatan, Bandar Sunway, 47500 Selangor Darul Ehsan, Malaysia.

${ }^{\mathrm{e}}$ Tropical Medicine and Biology Multidisplinary Platform, Monash University Malaysia, Jalan Lagoon Selatan, Bandar Sunway, 47500 Selangor Darul Ehsan, Malaysia.

${ }^{\mathrm{f}}$ Bornean Sun Bear Conservation Centre, Jalan Sepilok, Mile 14, Sandakan, 90000 Sabah, Malaysia.

${ }^{g}$ National Wildlife Forensic Laboratory (NWFL), Ex-situ Conservation Division, Department of Wildlife and National Parks (DWNP), KM 10 Jalan Cheras, 56100 Kuala Lumpur, Malaysia.

*Corresponding author email: Shyamala Ratnayeke, shyamalar@sunway.edu.my 


\begin{abstract}
Sun bear populations are fragmented and at risk from habitat loss and exploitation for body parts. These threats are made worse by significant gaps in knowledge of sun bear population genetic diversity, population connectivity, and taxonomically significant management units. Using a complete sun bear mitochondrial genome, we developed a set of mitochondrial markers to assess haplotype variation and the evolutionary history of sun bears from Peninsular (West) Malaysia, and Sabah (East Malaysia). Genetic samples from 28 sun bears from Peninsular Malaysia, 36 from Sabah, and 18 from Thailand were amplified with primers targeting a $1,800 \mathrm{bp}$ region of the mitochondrial genome including the complete mitochondrial control region and adjacent genes. Sequences were analyzed using phylogenetic methods. We identified 51 mitochondrial haplotypes among 82 sun bears. Phylogenetic and network analyses provided strong support for a deep split between Malaysian sun bears and sun bears in East Thailand and Yunnan province in China. The Malaysian lineage was further subdivided into two clades: Peninsular Malaysian and Malaysian Borneo (Sabah). Sun bears from Thailand occurred in both Sabah and Peninsular Malaysian clades. Our study supports recent findings that sun bears from Sundaland form a distinct clade from those in China and Indochina with Thailand possessing lineages from the three clades. Importantly we demonstrate a more recent and clear genetic delineation between sun bears from the Malay Peninsula and Sabah indicating historical barriers to gene flow within the Sundaic region.
\end{abstract}

Keywords: forest-dependent species, Sunda, Helarctos malayanus, subspecies, mtDNA 


\section{INTRODUCTION}

The distribution and population genetic structure of a species can provide useful insights about unique conservation units and landscape linkages that have facilitated historic and recent gene flow among populations (Veron et al. 2015; Patel et al. 2017). Populations may be adapted to different environmental conditions justifying management as separate units and decisions as to potential sites for reintroduction or repatriation (Palsbøll et al. 2007). Likewise, molecular data can aid in identifying the likely origins of confiscated animals or forensic samples, thereby indicating where wildlife law-enforcement efforts should be directed.

The Malayan sun bear (Helarctos malayanus) is native to Southeast Asia. Throughout their range, sun bear populations are at risk from the rapid conversion of rainforests to commercial plantations and from illegal exploitation for body parts that have a high market value (Shepherd and Shepherd 2010; Scotson et al. 2017a; Gomez et al. 2020). The previous distribution range of the sun bear is now greatly fragmented. Sun bears are currently listed as Vulnerable (IUCN 2019) with population trajectories based on rates of forest loss (Scotson et al. 2017b).

The reconnection of isolated populations of sun bears is a major goal of the recent status review and action plan for sun bears (Crudge et al. 2019). Kunde et al. (2019) reported extremely low levels of genetic diversity and a high inbreeding coefficient in a fragmented population of sun bears in Western Cambodia, highlighting the negative consequences of population isolation and barriers to dispersal. Population-level studies employing genetic samples from wild sun bears have been impeded by difficulties of capturing sun bears in the wild. Sun bears possess short hairs that do not snag on traditional barbed wire hair traps (e.g., Woods et al. 1999), but recent developments in non-invasive sampling have overcome this hurdle (Tee et al. 2020, in press). Developing mitochondrial markers that would provide sufficient variability to assess population genetic structure, and that could successfully amplify samples from hair root bulbs, would propel future research efforts on population estimation, intra- and inter-population diversity, gene flow and population connectivity in this imperiled species.

In this study, we developed mitochondrial markers to conduct the first molecular genetic assessment of the two subspecies of sun bear that occur in Western Peninsular Malaysia and 
Sabah in Malaysian Borneo. H. m. malayanus occurs on the Southeast Asian mainland, including parts of Sundaland, namely Peninsular Malaysia and Sumatra (Scotson et al. 2017a). The single subspecies, H. m. euryspilus, (Chasen 1940; Meijaard 2004) is found on the Sundaland island, Borneo, which includes the states of Sabah and Sarawak, East Malaysia. Recent evidence using whole mitochondrial genomes challenges the current subspecies delineation for sun bears, indicating a mid-Pleistocene split between Sundaland sun bears (i.e., Peninsular Malaysia, Sumatra and Borneo), and bears from China and Indochina (Kunde 2017). However, samples from Sundaland were few, including only two individuals from Peninsular Malaysia and one from Borneo.

The islands of the Sunda Shelf support a rich mammalian fauna whose distribution has been shaped by climate-induced changes in sea level that periodically exposed the Sunda Shelf or flooded it during interglacial periods (Heaney 1984). The Isthmus of Kra on the Thai-Malay peninsula represents a major climatic transition between the Indochina and Sundaic zoogeographic regions, including marked shifts in floral and faunal compositions (Woodruffe and Turner 2009; Hall 2012), but growing evidence from molecular and palaeoenvironmental studies suggests that species dispersal within Sundaland itself was restricted by geographic and ecological barriers during glacial periods (e.g., Veron et al. 2015; Patel et al. 2017; Wurster et al. 2020).

Our goal was to investigate haplotype diversity and phylogenetic relationships among sun bears from two parts of Sundaland: Peninsular Malaysia, and the Malaysian state of Sabah, Borneo. Using genetic samples from 64 sun bears from these two regions, we targeted a $\sim 1800$ bp region encompassing the control region of the sun bear mitochondrial genome, to evaluate genetic structure in these two populations and to compare their relationship with sun bears from the mainland. We included 18 sun bears from Thailand, which borders Peninsular Malaysia at the Isthmus of Kra, to evaluate evolutionary relationships between Malaysian bears and the adjacent mainland populations.

\section{METHODS}

Genomic DNA was extracted from 28 sun bears from West Malaysia, 36 sun bears from Sabah and 18 sun bears from Thailand using the Qiagen DNeasy Blood and Tissue Kit for tissue samples or blood collected on FTA cards, and the Qiagen Investigator Kit for hair or for blood preserved in EDTA (Supplementary Table 1). We included one Asiatic black bear 
(Ursus thibetanus) from Thailand and used the corresponding data to root the phylogenetic tree. Geographic locations were known for 19 bears from the peninsula and 29 from Sabah, but for only 4 bears from Thailand (two from East Thailand and two from West Thailand).

Using a recently sequenced sun bear mitochondrial genome (Lai Wai Ling unpublished), we designed three pairs of overlapping primers to target a region of approximately 1,800 bp of the mitochondrial genome including the entire mitochondrial control region (CR) and regions upstream and downstream of the CR: partial CYTB, tRNA-Thr, tRNA-Pro, tRNA-Phe and partial 12S rRNA (Supplementary Table 2, Supplementary Figure 1). We chose this region based on its well-known advantages for phylogenetic studies and within-species phylogeography: namely maternal inheritance, lack of recombination and fast evolutionary rates (Sunnucks 2000; Gupta et al. 2015).

\section{DNA extraction and PCR amplification}

Amplification of eluted DNA was performed using a Bio-Rad T100 Thermal Cycler PCR machine. PCR reaction solutions for mitochondrial markers CR1 and CR2 contained $30 \mathrm{ng}$ of DNA, $15 \mu \mathrm{L}$ of $2 \mathrm{X}$ ExPrime Taq Premix (with ExPrime Taq DNA Polymerase 1 unit / 10 $\mu \mathrm{L}, 2 \mathrm{X}$ reaction buffer, $4 \mathrm{mM} \mathrm{MgCl}_{2}$, enzyme stabilizer, sediment loading dye, $\mathrm{pH}$ 9.0, 0.5 $\mathrm{mM}$ of each dNTPs) (GeNet Bio, Korea), $1 \mu \mathrm{M}$ forward primer, and $1 \mu \mathrm{M}$ reverse primer in a total volume of $30 \mu \mathrm{L}$. The PCR cycling conditions started with an initial denaturation at 95 ${ }^{\circ} \mathrm{C}$ for 3 minutes, followed by 40 cycles of denaturation at $95{ }^{\circ} \mathrm{C}$ for 30 seconds, annealing at $55{ }^{\circ} \mathrm{C}$ for 30 seconds, extension at $72{ }^{\circ} \mathrm{C}$ for 1 minute and ending with a final extension at 72 ${ }^{\circ} \mathrm{C}$ for 10 minutes. For CR3, optimal amplification was obtained using $40 \mathrm{ng}$ DNA, $0.7 \mu \mathrm{M}$ forward and reverse primer with an annealing temperature of $65{ }^{\circ} \mathrm{C}$. Malaysian sun bear amplicons were sequenced using the NEBNext ${ }^{\circledR}$ Ultra $^{\mathrm{TM}}$ DNA Library Prep Kit (New England Biolabs Inc.) and the Illumina MiSeq platform (Illumina, San Diego, CA). The instrument was set to produce 1,000 reads per sample using 2 x 250 bp read length configuration to obtain $30 \mathrm{X}$ coverage. Amplicons for Thai samples were sequenced using Sanger sequencing. 


\section{Phylogenetic and haplotypic network analysis using Bayesian and Maximum Likelihood Trees}

The sequencing data was imported into Geneious v11.1.5 software (Biomatters Ltd., Auckland, New Zealand. Forward and reverse reads were paired and merged. A reference mitochondrial genome of a sun bear and an Asiatic black bear from Yunnan, China (GenBank accession nos. FM177765, EF196664) was trimmed to obtain the targeted amplification region. Merged reads from each sample were mapped to the trimmed reference genome to generate a consensus sequence. Consensus sequences from all Malaysian $(n=64)$ and Thailand sun bear samples $(n=18)$ were compiled into one file. Sequences were checked and edited manually using BioEdit 7.0.9.0 (Hall 1999) and aligned using Clustal X (Thompson et al. 1997).

We used sequence data for all 82 sun bears, including the Asiatic black bear sequence from Thailand, and used a maximum likelihood (ML) approach and Bayesian Inference (BI) to construct phylogenetic trees. For ML, the best-fitting model $(\mathrm{GTR}+\mathrm{G}+\mathrm{I})$ ) was estimated prior to analysis using jModelTest v2.1.1 and Akaike's Information Criterion (AIC; Akaike 1974). The selected model was implemented in ML analysis using MEGA 7 (Kumar et al. 2016) using 1000 bootstrap replicates to evaluate nodal support. The two Ursus thibetanus sequences from Yunnan and Thailand were used as an outgroup to root phylogenies and the reconstructed phylogenetic tree viewed in FigTree v.1.4.0 (Rambaut 2012).

We used BEAST v1.8 (Drummond et al. 2012) to estimate time to the most recent common ancestor for major nodes and to generate a BI phylogeny. The best fitting substitution model for the data was General Time Reversible $(\mathrm{GTR}+\mathrm{G}+\mathrm{I}$; determined using the model-fitting tool within MEGA7; Kumar et al. 2016). The root of the tree was fixed at 6 million years before present (BP), based on the divergence of $U$. thibetanus and $H$. malayanus (Kumar et al. 2017a). Tracer (Rambaut et al. 2018) determined that a strict clock and Bayesian skyline were the most likely combination of clock and tree parameters for the dataset.

We used MEGA7 and DNAsp v. 6 (Rozas et al. 2017) to compute haplotype diversity, nucleotide diversity, and mean pairwise genetic distances for Malaysian samples of known origin, and all Thai samples. We used Arlequin ver 3.5.2.2 (Excoffier and Lischer 2010) to compute genetic differentiation $\left(\mathrm{F}_{\mathrm{ST}}\right)$ among Peninsula, Sabah and Thai sequences. Mean pairwise genetic distances and AMOVA results were based on 10,000 permutations. 


\section{RESULTS}

Tree topologies generated by ML and BI analyses of the $\sim 1,800 \mathrm{bp}$ continuous sequence supported a deep split between sun bears from Thailand/Yunnan China (mainland) and Malaysian bears that occurred $\sim 1.5$ million years ago (MYA; Fig. 1). Two sun bears confirmed to originate from East Thailand occurred in this clade, including four additional Thai bears whose specific geographic origin within Thailand was unknown. Malaysian sun bears showed a more recent split ( 600,000 years ago) forming two major lineages, one from Peninsular Malaysia and one from Sabah (Fig. 1). One of two sun bears from West Thailand were found in each of these clades (T1 and T11). Ten additional sun bears from Thailand were also placed in the Peninsular and Sabah clades, but it is not known where in Thailand these bears originated.

Mitochondrial sequences of three sun bears, whose samples were obtained in the peninsula, clustered with Bornean bears. Wildlife authorities confiscated P17 and P15 from private owners in the Malay peninsula; P27 was from a local zoo. No details of the origins of P17 and P15 were available. P27 was reported to be the cub of female P22 and male P24, which was not supported by the sequence data.

The combined sequence revealed 51 haplotypes among 82 bears and 34 haplotypes among 48 samples from known geographic locations within Malaysia. Genetic differentiation among Sabah, Peninsula and Thai sun bears was significant and greatest between Sabah and Peninsula bears (Table 1). Genetic differentiation among Thai lineages was even greater. 
Table 1. A. Summary of sample numbers $(\mathrm{N})$, percent mean pairwise genetic distance $(D)$, haplotypes $(\mathrm{h})$, haplotype diversity $(\mathrm{Hd})$, and nucleotide diversity $(\pi)$ for bears from known geographic locations within Sabah and Peninsular Malaysia, and all Thai bears. B. Genetic differentiation $F_{\mathrm{ST}}$ among Peninsula Malaysia, Sabah, and Thai populations. C. Genetic differentiation $F_{\mathrm{ST}}$ among Thai sun bears in Peninsula Malaysia, Sabah, and mainland clades.

\begin{tabular}{|c|c|c|c|c|c|}
\hline Population(s) & $N$ & $D$ & $\mathbf{H}$ & $H_{\mathrm{d}}$ & $\pi$ \\
\hline \multicolumn{6}{|l|}{ A. Genetic Diversity } \\
\hline Sabah, Borneo & 29 & 0.86 & 22 & 0.966 & 0.006 \\
\hline Peninsular Malaysia & 19 & 0.61 & 12 & & 0.003 \\
\hline \multirow[t]{2}{*}{ Thailand } & 18 & 1.59 & 11 & & 0.013 \\
\hline & $N_{1} / N_{2}$ & $D$ & $F_{\mathrm{ST}}$ & & \\
\hline \multicolumn{6}{|l|}{ B. Genetic differentiation } \\
\hline Sabah - Peninsula & $29 / 19$ & 1.39 & $0.556 * * *$ & & \\
\hline Sabah - Thailand (all) & $29 / 18$ & 1.65 & $0,410 * * *$ & & \\
\hline \multirow[t]{2}{*}{ Peninsula - Thailand (all) } & $19 / 18$ & & $0.363 * * *$ & & \\
\hline & $N_{1} / N_{2}$ & $D$ & $F_{S T}$ & & \\
\hline \multicolumn{6}{|l|}{ C. Genetic differentiation - Thailand } \\
\hline Thai Sabah - Thai Peninsula & $6 / 6$ & 1.46 & $0.467 * * *$ & & \\
\hline Thai Sabah - Thai mainland & $6 / 6$ & 2.47 & $0.690 * * *$ & & \\
\hline Thai Peninsula - Thai mainland & $6 / 6$ & 2.37 & $0.470 * * *$ & & \\
\hline
\end{tabular}

\section{DISCUSSION}

We characterized mitochondrial variation by targeted resequencing $\sim 1,800$ bp of the mitochondrial CR in 82 sun bears from Malaysia and Thailand. Our results suggest a midPleistocene divergence of sun bears in mainland Southeast Asia into two lineages, of which one lineage diversified in Sundaland. Moreover, we provide the first molecular evidence that sun bears in Sabah/Borneo form a clade distinct from those on the Malay peninsula. Western Thai sequences were mixed in with both peninsula and Sabah sequences. Since the only reliable calibration point for our Bayesian tree was the divergence of Helarctos malayanus and Ursus thibetanus at $6 \mathrm{MYA}$, which occurred long before the diversification within $H$. malayanus, we acknowledge considerable uncertainty about when these divergences 
occurred, but our results confirm Kunde's (2017) findings of an ancient split in the sun bear lineage on the Asian mainland, one lineage diversifying in the Sundaic region and the other on the Asian mainland. Also, in this study, haplotypes from Thailand occurred in both lineages. An older common ancestry to Yunnan and Malaysian (peninsular and Bornean) bears, including a more recent divergence of peninsular and Bornean bears suggests that sun bears first evolved on the Asian mainland (Louys 2012) rather than in Sundaland as previously hypothesized (Meijaard 2004).

A possible explanation for the patterns in our study is that sun bears spread southwards from Indochina in the mid-Pleistocene, during glacial maxima when sea levels were low and the Sunda shelf was exposed as a contiguous landmass (Figure 2A). During interglacial periods when sea levels rose and covered most of the Sunda shelf, populations differentiated in the Malay peninsula and Malaysian Borneo. During subsequent glacial events that exposed the Sunda shelf once again, bears migrated northwards from both Malaysian areas to Western Thailand (Figure 2B). We posit that Thai clades within Malaysian lineages were most likely derived from Peninsula and Bornean clades that diverged much earlier, at around 600,000 yrs. Had those same Thai sun bear lineages been derived from populations elsewhere, deeper origins in the tree would be expected.

A second explanation is that deep bifurcation occurred in Thailand followed by southward migration, far later than the bifurcation, e.g. just before the Last Glacial Maximum, after considerable post-bifurcation differentiation in Sundaland had occurred. This late migration scenario is less likely because there is little evidence of mixing between peninsula and Sabah lineages. Although three bears from the Malaysian Peninsula appear in the Bornean clade, their geographic origins were uncertain. The lack of confirmed peninsula haplotypes in the Bornean clade suggests that the northward movement of bears to Western Thailand did not overlap the peninsula, at least in recent times. Palaeoecological evidence suggests marked climatic and vegetational shifts accompanying glacial-interglacial periods, with the habitat of the Malay peninsula shifting from open woodlands during glacial periods, to closed deciduous and evergreen rain forest during interglacial periods (Louys and Meijaard 2010). Wurster et al. (2020) provide evidence of a north-south savanna corridor from peninsular Malaysia, through central Sundaland that existed for much of the last glacial period (110 $11.7 \mathrm{ka}$ ) that would have restricted dispersal of forest-dependent species such as sun bears. Such a barrier could have contributed to east-west differentiation of sun bear populations in Sundaland and the clear differentiation of Sabah and Peninsular Malaysia clades. Also, a 
primarily northward movement, after differentiation in Sundaland, would explain the morphological uniqueness of Bornean sun bears (Horsfield 1825; Meijaard 2004) whose populations would have remained relatively isolated since initial colonization.

Our data suggest that Thailand contains at least three well-differentiated mitochondrial lineages (Yunnan/Indochinese origin, Sabah/Borneo ancestry, and peninsula ancestry) and two populations: an Eastern Thailand population consisting of the Yunnan/Indochinese lineage, and a Western Thailand population consisting of lineages from the peninsula and Borneo. It could be argued that the two Malaysian lineages are isolated remnants of a larger pool of ancestral haplotype diversity retained in Thailand, but Malaysian haplotype diversity and tree topology suggest otherwise. Thai lineages within peninsula and Sabah clades appear to be recently evolved from Malaysian ancestors, whereas divergence between peninsula and Sabah lineages began much earlier, after geographic isolation. The proposed differentiation among Thai lineages is limited by the fact that we have only two confirmed west Thai sequences (T1 in the Sabah clade and T11 in the peninsula clade), and two confirmed east Thai sequences (T7 and T12) in the mainland clade. The back-migration hypothesis is also based on a limited number of sequences from Thailand. More sequences of nuclear and mitochondrial sources from known geographic locations in Thailand, Borneo, and Sumatra, will help to refine our hypotheses and elucidate the phylogenetic history of sun bears in this region. Even though the control region is recognized as the fastest evolving part of the mitochondrion, we think that mutational saturation or long branch attraction (LBA) is unlikely to have influenced phylogenetic inference. Over the timescale of the study, only 92 out of 1,968 bp positions in the alignment showed any signs of variation. The number of substitutions between our two in-group clades (Sabah and Peninsula) was only 25 on average and never more than 36 . The average number of substitutions between our in-groups and the east Thai out-group was only 41. By contrast, T11 (west Thai) differed from P22 (Peninsula) by only four substitutions and T1 (west Thai) by only 9 substitutions from B13.

Considering the current mitochondrial DNA phylogenetic evidence of a deep historical separation between sun bears in Sundaland from bears on mainland Southeast Asia, a reexamination of the subspecies designations, H. m. malayanus and $H . m$. euryspilus, is warranted. The name Helarctos annamiticus, Heude 1901, was given to 3 skulls from Vietnam, based on differences in cranial and dental features compared to Sumatran sun bears. However, Pocock (1941) found insufficient evidence to consider H. annamiticus a distinctive form or local race. Body size, skull and dental characteristics of the Bornean sun bear are 
distinctive (Horsefield 1925, Pocock 1941, Chasen 1940). Craniometric analyses of 34 sun bears from Borneo, Sumatra, and the Asian mainland (including the Malay peninsula) indicate significantly smaller cranial, but relatively larger maxillary tooth dimensions in Bornean sun bears (Meijaard 2004). No significant differences were detected between Sumatran and mainland bears supporting the designation of two subspecies: $H$. $m$ euryspilus (Borneo) and H. $m$ malayanus (Sumatra and the mainland). However, 4 of the 9 mainland individuals in this study were from Peninsular Malaysia. Our data suggest why Bornean populations may indeed be unique, but generates questions about relationships among Sumatran, peninsular, and mainland populations.

The maternally inherited nature of mitochondrial genes may obscure true phylogenetic relationships in species such as ursids that tend to exhibit male-biased dispersal and interspecific gene flow (Kumar et al. 2017b). Thus, inferences of population history based purely on mitochondrial DNA may be limited or misleading due to incomplete lineage sorting or introgression of nuclear genes (Moritz 1994; Zhang and Hewitt 2003; Funk and Omland 2003; Kutschera et al. 2014). A more complete understanding of the phylogenetic and population structure of sun bears may be achieved by the inclusion of several nuclear and mitochondrial markers (Rubinoff and Holland 2005) or whole genome sequencing. Despite these caveats, this study provides an important step toward elucidating the phylogenetic history of sun bears in Peninsular Malaysia and Sabah. Furthermore, our results highlight potential gaps in our knowledge of evolutionarily significant units in this species and the need for morphogenetic analyses using specimens across the entire distribution range.

The high haplotype diversity detected in this study reflects the high differentiating power of the mitochondrial DNA markers used. They may be applicable, therefore, to forensic studies where the origin of confiscated or surrendered bears is of interest, or for identifying potential sites of release for reintroduction programs. Apart from targeting highly polymorphic regions of the mitochondrial genome, the primers used in this study may be effective for samples where poor DNA quality and quantity precludes whole mitogenome construction. We successfully amplified all hair and blood samples in this study, including 20 hair samples collected non-invasively from wild sun bears using the CR1 (764 bp) marker (Tee et al. 2020). These markers hold much promise, therefore, for studies of genetic isolation and contemporary gene flow in sun bear populations. 


\section{Funding}

This work was supported by the Sunway Internal Grant (INT-FST-BIOS-0115-02) funded by Sunway University, Malaysia, and the International Association for Bear Research and Management (IBA).

\section{Acknowledgements}

We thank Khannamthong Phunnoi, Phatchamon Boonprasong and Sunita Wingwon for help with collecting hair samples from Thai bears, Tee Thye Lim for samples from Sabah, and Eve Foong Yee Wei for samples from Sunway Lagoon Wildlife Park. Suganiya Rama Rao assisted with bioinformatics and data analysis.

\section{Data Availability}

We have deposited the primary data underlying these analyses as follows:

- Mitochondrial sequences: GenBank accession MW316360-MW316405.

- Sampling locations, haplotypes, and collection details are uploaded online Supplementary Table 1.

- Primer sequences, positions and expected amplicon lengths of the three pairs of overlapping mitochondrial markers are in Supplementary Table 2, Figure 1. 


\section{References}

Akaike H. 1974. A new look at the statistical model identification. IEEE Trans Autom Control. 19:716-723.

Chasen FN., 1940. A Handlist of Malaysian Mammals- A Systematic List of the Mammals of the Malay Peninsula, Sumatra, Borneo and Java, Including the Adjacent Small Islands. Bulletin of the Raffles Museum 15.

Crudge B, Lees C, Hunt M, Steinmetz R, Fredriksson G, Garshelis D. 2019. Sun bears: global status review and conservation action plan, 2019-2028. IUCN SSC Bear Specialist Group/IUCN SSC Conservation Planning Specialist Group/Free the Bears/TRAFFIC.

Excoffier L, Lischer HEL. 2010. Arlequin suite ver 3.5: A new series of programs to perform population genetics analyses under Linux and Windows. Mol Ecol Res. 10:564-567.

Funk DJ, Omland KE. 2003. Species-level paraphyly and polyphyly: Frequency, causes, and consequences, with insights from animal mitochondrial DNA. Ann Rev Ecol Syst. 34: $397-423$.

Gomez L, Shepherd CR, Khoo MS. 2020. Illegal trade of sun bear parts in the Malaysian states of Sabah and Sarawak. Endanger Species Res. 41:279-287.

Gupta A, Bhardwaj A, Sharma P, Pal Y. 2015. Mitochondrial DNA-a tool for phylogenetic and biodiversity search in equines. Journal of Biodiversity \& Endangered Species S1: S1.006. doi:10.4172/2332-2543.S1-006.

Hall R, 2012. Sundaland and Wallacea: geology, plate tectonics and palaeogeography. In: Gower DJ, Johnson K, Richardson J, Rosen B, Rüber L, Williams S, editors. Biotic evolution and environmental change in Southeast Asia. Cambridge University Press. p. 33-78.

Hall TA. 1999. BioEdit: a user-friendly biological sequence alignment editor and analysis program for Windows 95/98/NT. Nucleic acids symposium series. [London]: Information Retrieval Ltd, c1979-c2000. p. 95-98.

Heaney LR. 1984. Mammalian species richness on islands on the Sunda Shelf, Southeast Asia. Oecologia, 61(1):11-17. 
Horsfield T. 1825. Description of the Helarctos Euryspilus; Exhibiting in the Bear from the Island of Borneo, the Type of a Subgenus of Ursus. The Zoological Journal (London) 2: $221-34$.

Kearse M, Moir R, Wilson A, Stones-Havas S, Cheung M, Sturrock S, Buxton S, Cooper A, Markowitz S, Duran C. 2012. Geneious Basic: an integrated and extendable desktop software platform for the organization and analysis of sequence data. Bioinform. 28:1647-1649.

Kunde MN. 2017. Ex-situ and In-situ Conservation Approach for the Malayan sun bear (Helarctos malayanus). Thesis (PhD Doctorate). Griffith University, Brisbane.

Kunde MN, Martins RF, Premier J, Fickel J, Forster DW. 2019. Population and landscape genetic analysis of the Malayan sun bear. Conserv Genet. doi.org/10.1007/s10592-01901233-w.

Kumar S, Stecher G, Tamura K. 2016. MEGA7: Molecular Evolutionary Genetics Analysis Version 7.0 for Bigger Datasets. Mol Biol Evol. 33:1870-1874.

Kumar S, Stecher G, Suleski M, Hedges SB. 2017a. TimeTree: a resource for timelines, timetrees, and divergence times. Mol Biol Evol. 34:1812-1819.

Kumar V, Lammers F, Bidon T, Pfenninger M, Kolter L, Nilsson MA, Janke A. 2017b. The evolutionary history of bears is characterized by gene flow across species. Sci Rep, 7: 46487. doi:10.1038/srep46487

Kutschera VE, Bidon T, Hailer F, Rodi JL, Fain SR, Janke A. 2014. Bears in a forest of gene trees: phylogenetic inference is complicated by incomplete lineage sorting and gene flow. Mol Biol Evol. 31:2004-2017.

Louys J. 2012. The future of mammals in Southeast Asia: conservation insights from the fossil record. In: Louys J, editor. Paleontology in Ecology and Conservation. Springer. p. $227-238$.

Meijaard E. 2004. Craniometric differences among Malayan sun bears (Ursus malayanus); evolutionary and taxonomic implications. Raffles Bulletin of Zoology 52:665-672.

Moritz C. 1994. Defining ‘evolutionarily significant units' for conservation. Trends Ecol Evol. 9:373-375.

Palsbøll PJ, Berube M, Allendorf FW. 2007. Identification of management units using population genetic data. Trends Ecol Evol. 22:11-16.

Patel RP, Wutke S, Lenz D, Mukherjee S, Ramakrishnan U, Veron G, Fickel J, Wilting A, Förster DW. 2017. Genetic structure and phylogeography of the leopard cat 
(Prionailurus bengalensis) inferred from mitochondrial genomes. J Hered. 108(4):349360. doi:10.1093/jhered/esx017.

Pocock RI. 1941. Family Ursidae. In: Sewell RBS, editor. The Fauna of British India, Including Ceylon and Burma. Taylor and Francis, p. 163-232.

Rambaut A. 2012. Rambaut A. FigTree v.1.4.2: Tree Figure Drawing Tool. Available online at: http://tree.bio.ed.ac.uk/software/figtree (Accessed Sep 30, 2020).

Rambaut A, Drummond AJ, Xie D, Baele G, Suchard MA. 2018. Posterior Summarization in Bayesian Phylogenetics Using Tracer 1.7. Syst Biol. 67(5):901-904. doi: 10.1093/sysbio/syy032. PMID: 29718447; PMCID: PMC6101584.

Rozas J, Ferrer-Mata A, Sánchez-DelBarrio JC, Guirao-Rico S, Librado P, Ramos-Onsins SE, Sánchez-Gracia A. 2017. DnaSP v6: DNA sequence polymorphisms of large datasets. Mol Biol Evol. 34:3299-3302.

Rubinoff D, Holland BS. 2005. Between two extremes: mitochondrial DNA is neither the panacea nor the nemesis of phylogenetic and taxonomic inference. Syst Biol. 54(6):952961.

Scotson L, Fredriksson G, Augeri D, Cheah C, Ngoprasert D, Wai Ming W. 2017a. Helarctos malayanus. The IUCN Red List of Threatened Species 2017. e.T9760A123798233. http://dx.doi.org/10.2305/IUCN.UK.2017-3.RLTS.T9760A45033547.en. <Accessed 28 Jul 2020>

Scotson L, Fredriksson G, Ngoprasert D, Wong WM, Fieberg J. 2017b. Projecting rangewide sun bear population trends using tree cover and camera-trap bycatch data. PLoS One 12(9):e0185336. https://doi.org/10.1371/journal.pone.0185336.

Shepherd CR, Shepherd L. 2010. The poaching and trade of Malayan sun bears in Peninsular Malaysia. Traffic Bulletin 23:49-52.

Sunnucks P. 2000. Efficient genetic markers for population biology. Trends in Ecology \& Evolution 15:199-203.

Tee TT, Lai WL, Kok TJW, Ooi ZH, van Manen FT, Sharp SP, Wong ST, Chew J, Ratnayeke S. 2020. An evaluation of non-invasive sampling techniques for Malayan sun bears. Ursus, 2020(31e16):1-12 (2020). https://doi.org/10.2192/URSUS-S-2000004.1.

Thompson JD, Gibson TJ, Plewniak F, Jeanmougin F, Higgins DG. 1997. The CLUSTAL_X windows interface: flexible strategies for multiple sequence alignment aided by quality analysis tools. Nucleic Acids Res. 25:4876-4882. 
Veron G, Patou ML, Jennings AP. 2015. Molecular systematics of the small-toothed palm civet (Arctogalidia trivirgata) reveals a strong divergence of Bornean populations. Mamm Biol. 80(4):347-354.

Woods JG, Paetkau D, Lewis D, McLellan BL, Proctor M, Strobeck C. 1999. Genetic tagging free-ranging black and brown bears in mark-recapture experiments. Wildl Soc Bull. 27: 616-27.

Wurster CM, Rifai H, Zhou B, Haig J, Bird MI. 2019. Savanna in equatorial Borneo during the late Pleistocene. Sci Rep. 9(1):1-7.

Zhang DX, Hewitt GM. 2003. Nuclear DNA analyses in genetic studies of populations: practice, problems and prospects. Mol Ecol. 12:563-584 


\section{LIST OF FIGURES}

Figure 1. Bayesian tree of sun bear divergence with Ursus thibetanus as the outgroup and time in millions of years ago (MYA) on the horizontal axis. Branches are colour-coded according to predicted region. Support values for major nodes represent Bayesian posterior probabilities/\% bootstraps obtained from maximum likelihood analyses. The root of the tree was fixed at 6 MYA based on an estimated divergence of $U$. thibetanus and Helarctos malayanus (http://timetree.org). Sun bears from Western Thailand occurred in both Peninsular Malaysia and Sabah clades. Prefixes P, B and T correspond to samples provided from the peninsula, Sabah/Borneo, and Thailand with district/state of origin stated where known. Sun bears from East Thailand shared a clade with a sun bear from Yunnan province, China. Confiscated/unknown refers to three samples obtained in the peninsula, but peninsula origin could not be confirmed. See online version for full colors.

Figure 2. Hypothesized movement of sun bears into and out of Sundaland based on phylogenetic data: A) southward from the East Thailand/Indochinese mainland to Sundaland, during the mid- Pleistocene, leading to initial colonization of Sabah and the Malay peninsula, and B) back-migration to west Thailand during subsequent glacial (or interglacial) events. Lighter areas of the Sunda shelf ( $<120 \mathrm{~m}$ depth) would have been exposed for long periods during the last $2.5 \mathrm{My}$. Country boundaries sourced from Global Administrative Areas (2012). GADM database of Global Administrative Areas, version 2.0. [online] URL: www.gadm.org. Bathymetric contours grid sourced from GEBCO Compilation Group (2020) GEBCO 2020 Grid (doi:10.5285/a29c5465-b138-234d-e053-6c86abc040b9). See online version for full colors.

\section{LIST OF TABLES}

Table 1. A. Summary of sample numbers $(\mathrm{N})$, percent mean pairwise genetic distance $(D)$, haplotypes (h), haplotype diversity (Hd), and nucleotide diversity $(\pi)$ for bears from known geographic locations within Sabah and Peninsular Malaysia, and all Thai bears. B. Genetic differentiation $F_{\mathrm{ST}}$ among Peninsula Malaysia, Sabah, and Thai populations. C. Genetic differentiation $F_{\text {ST }}$ among Thai sun bears in Peninsula Malaysia, Sabah, and mainland clades. 
Figure 1

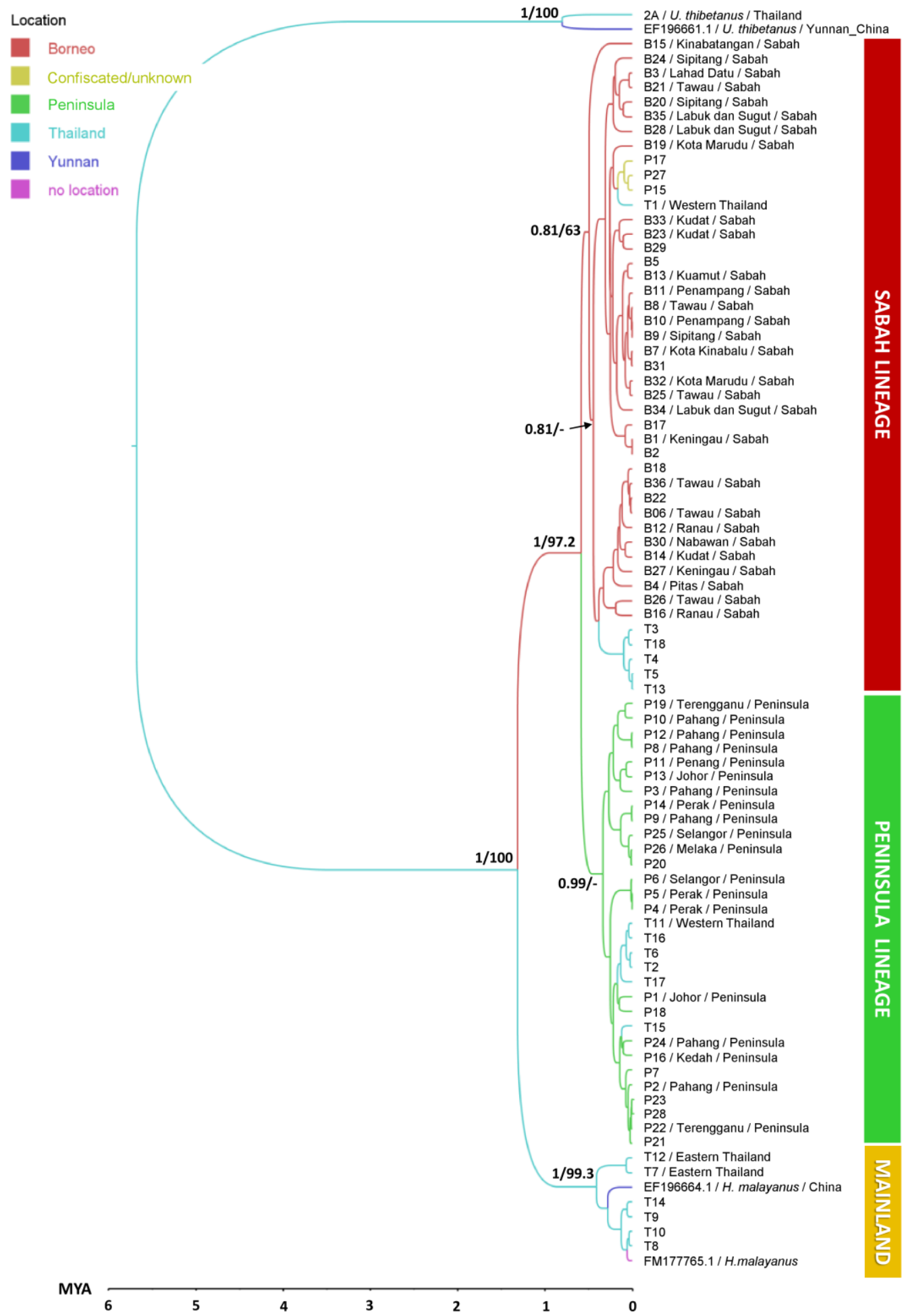


Figure 2
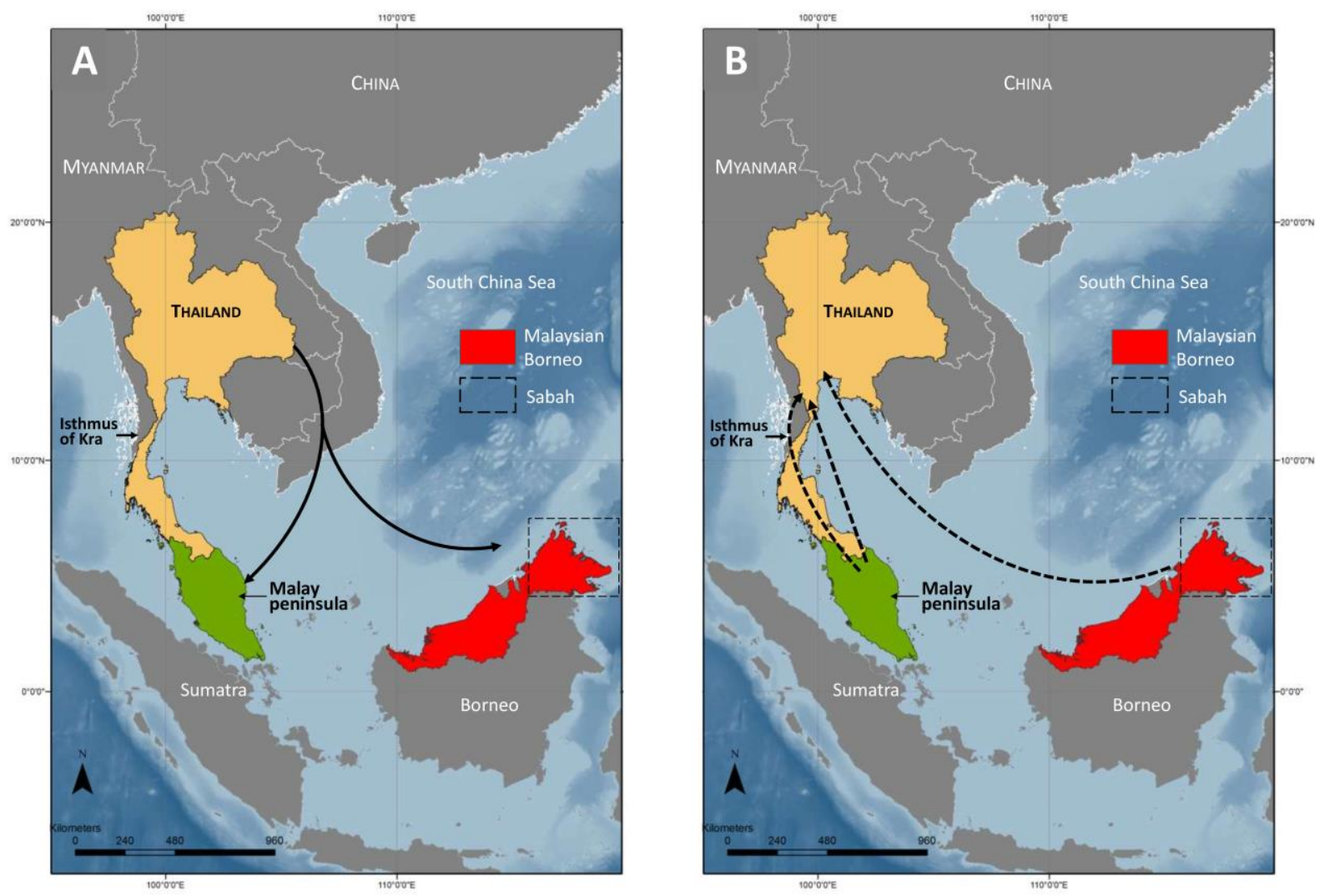